\title{
Compartilhamento de conhecimento: estudo bibliométrico das publicações acadêmicas realizadas de 1994 a 2014
}

Marilei Osinski

\begin{abstract}
Bacharela em Ciências da Administração pela Universidade Federal de Santa Catarina. Mestranda em Engenharia e Gestão do Conhecimento na Universidade Federal de Santa Catarina
\end{abstract}

Darlan José Roman

Doutor em Administração pela Universidade Federal de Santa Catarina. Professor da Universidade do Oeste de Santa Catarina

Paulo Mauricio Selig

Doutor em Engenharia de Produção pela Universidade Federal de Santa Catarina Professor da Universidade Federal de Santa Catarina

http://dx.doi.org/10.1590/1981-5344/2512

Atualmente o conhecimento é considerado um dos principais fatores de produção e agregação de valor, tanto no meio acadêmico quanto organizacional. A Gestão do Conhecimento, por sua vez, é considerada uma forma de melhorar o desempenho e aumentar a competitividade. Diante desses fatos, o objetivo da presente pesquisa foi fazer uma bibliometria dos artigos que tratam do tema compartilhamento de conhecimento no período de 1994 a 2014, disponíveis nas bases de dados SciELO e Scopus. Para alcançar o objetivo, inicialmente foi feita uma busca dirigida nas bases de dados em questão, selecionando os artigos pertinentes, que foram analisados em seguida. $A$ presente pesquisa é quali-quantitativa e classifica-se como bibliométrica, descritiva, indutiva, aplicada e bibliográfica. Dentre os principais resultados alcançados percebe-se um crescimento expressivo do número de publicações a respeito de Compartilhamento de conhecimento no período analisado.

Palavras-chave: Gestão do conhecimento. Compartilhamento de conhecimento. Bibliometria. Ativos intangíveis. Competitividade. 


\section{Sharing knowledge: bibliometric study of academic publications held from 1994 to 2014}

The knowledge is considered currently as a major factor of production and value added, both in academic and organizational environment. The Knowledge Management is a way to improve performance and increase competitiveness. The objective of this research was to make a bibliometrics study about the articles dealing with the issue of knowledge sharing in the 1994-2014 period, available in the databases Scopus and SciELO. To achieve the goal, initially directed a search in the databases in question, selecting relevant articles, which were then analyzed was taken. This research is qualitativequantitative and ranks as bibliometrics, descriptive, inductive, applied and bibliographic. Among the main results stands out a significant increase in the number of publications about knowledge sharing in the analyzed period.

Keywords: Knowledge management. Knowledge sharing. Bibliometrics. Intangible assets. Competitiveness.

Recebido em 17.08.2015 Aceito em 20.11.2015

\section{Introdução}

As organizações estão inseridas num ambiente complexo e que sofre mudanças constantes. Adaptar-se, evoluir e inovar são ações essenciais para que as empresas mantenham-se competitivas na atual configuração do mercado. Nesse meio, um dos principais fatores de diferenciação organizacional, agregação de valor e destaque entre os concorrentes é o conhecimento. Assim, manter e valorizar ativos intangíveis pode ser uma forma de ampliar vantagens competitivas em relação aos concorrentes (TONET; PAZ, 2006; RAMOS; HELAL, 2010; CUNHA; FERREIRA, 2011; FREIRE et al., 2012; LEMOS; JOIA, 2012).

Uma alternativa interessante apontada pelos autores estudados nessa pesquisa para manter o conhecimento organizacional é o compartilhamento de conhecimento. O compartilhamento de conhecimento busca a transmissão e sinergia de conhecimentos e experiências de um indivíduo (funcionário) para outro, oferecendo a possibilidade de ampliar conhecimentos. Diante da necessidade de manter o máximo de conhecimento possível no cenário competitivo atual, o 
objetivo desta pesquisa foi fazer uma bibliometria dos artigos que tratam do tema compartilhamento de conhecimento no período de 1994 a 2014, disponíveis nas bases de dados SciELO e Scopus.

Após esta seção de introdução este trabalho apresenta outros tópicos que compõe a estrutura da pesquisa. A segunda etapa apresentada consiste no referencial teórico, tratando dos seguintes temas: Gestão do Conhecimento e compartilhamento de conhecimento. Após o referencial teórico é apresentada a metodologia, e na seção seguinte é apresentada a análise e discussão dos dados. Finalmente, é apresentada a conclusão, seguida pelas referências utilizadas na realização desse estudo.

\section{Gestão do conhecimento}

O termo "Gestão do Conhecimento" tem dominado o vocabulário tanto de pesquisadores quanto de empresários e gestores ao longo da história. Apesar da popularização crescente no meio acadêmico e empresarial, não existe entre os teóricos, até o momento presente, um consenso acerca do conceito do tema em questão (OLIVEIRA et al., 2013). No entanto, apesar da variedade de conceitos disponível, é consenso que a Gestão do Conhecimento pode ser considerada uma forma eficiente para manter os ativos intelectuais da estrutura organizacional (TORRES; ZIVIANI; SILVA, 2012).

Conforme Cunha; Ferreira (2011) o conhecimento é um forte fator de diferenciação organizacional e destaque dentre os concorrentes. A Gestão do Conhecimento pode gerar vantagem competitiva sustentável, pois é possível que duas pessoas, ao compartilharem seus conhecimentos individuais combinem ambos para gerar outro conhecimento inédito, diferente daquele(s) que detinham anteriormente. O efeito causado pela sinergia desse processo faz com que o conhecimento resultante dessa junção de conhecimentos seja maior que a soma dos conhecimentos individuais.

A Gestão do Conhecimento é uma alternativa para, além de manter ativos intangíveis, ampliar vantagens competitivas em relação aos concorrentes. O conhecimento torna-se, cada vez mais, um dos fatores mais importantes para a competitividade organizacional (FREIRE et al., 2012). No mesmo sentido, Binotto, Nakayama e Siqueira (2013) afirmam que uma organização que cria conhecimentos pode ser considerada um sistema aberto, buscando adequar-se constantemente, garantindo sua sobrevivência e certeza de competitividade no mercado.

Reforçando o pensamento de Freire et al. (2012); Binotto, Nakayama e Siqueira (2013) de que o conhecimento é um dos fatores mais importantes para a competitividade organizacional atualmente, Evers (2001) afirma que o conhecimento passa a ser considerado o principal fator de produção, agregação de valor e crescimento econômico no cenário atual. Então, gerenciar o conhecimento torna-se uma das atividades mais importantes no contexto organizacional. 
Complementando esse raciocínio, Tonet e Paz (2006) destacam que o conhecimento é um recurso fundamental para as organizações, tanto públicas quanto privadas, e que as mesmas precisam entender as próprias necessidades de descobrir o que sabem, e usar esse saber. A Gestão do Conhecimento é um processo que busca otimizar o uso desse recurso intangível e que tem, como uma de suas etapas e/ou objetivos, o compartilhamento de conhecimento.

Concordando com as afirmações de Tonet e Paz (2006); Ramos e Helal (2010); Cunha e Ferreira (2011); Freire et al. (2012), entre outros, Lemos e Joia (2012), inferem que o conhecimento é estudado há bastante tempo na área de Gestão. Porém, a partir de 1990, impulsionadas pelo crescimento da economia baseada em ativos intangíveis, as organizações passaram a preocupar-se, de forma mais expressiva, com o conhecimento existente no próprio ambiente de trabalho - ou seja, nas organizações em si - e com o gerenciamento desse conhecimento. Assim, a Gestão do Conhecimento foi impulsionada, conquistando espaços no meio empresarial.

Dentre os aspectos principais da Gestão do Conhecimento pode-se destacar o fato de lidar, sobretudo, com bens intangíveis da organização. A Gestão do Conhecimento busca organizações capazes de compreender o ambiente no qual estão inseridas, bem como suas necessidades. Nesse processo é necessária a presença de colaboradores cada vez mais eficientes, capazes de compreender a rapidez das informações e a necessidade de renovação constante. Os ativos intangíveis estão sendo valorizados de forma constante e crescente ao longo do tempo no ambiente das organizações e dos negócios, uma vez que o interesse pelo conhecimento aumenta à medida que seu valor é percebido (RAMOS; HELAL, 2010).

O interesse pelo conhecimento é exemplificado por Tonet e Paz (2006), que descrevem a constatação do fato de que o valor de mercado de determinadas empresas é inúmeras vezes maior que o valor do patrimônio financeiro e/ou físico que possuem. Nesses casos, as ações valem tanto porque incorporam valores intangíveis - como marca, capacidade de inovação, talento e competência dos empregados, entre outros. A maior parte do valor intangível é agregada pela posse de conhecimento, resultado da incorporação de novas experiências e aprendizagem contínua e compartilhada. Nesse âmbito, contar com uma equipe de Gestão do Conhecimento capacitada, competitiva e atualizada é fundamental para o sucesso organizacional.

\subsection{Compartilhamento de conhecimento}

Diversas nomenclaturas são utilizadas atualmente para descrever o processo de compartilhamento de conhecimento. As expressões comumente utilizadas são "transferência de conhecimento", "compartilhamento de conhecimento", "disseminação de conhecimento", 
"transmissão de conhecimento", "difusão de conhecimento", "troca de conhecimento", dentre outras, que podem ser consideradas sinônimos. Autores como Ferreira, Li e Serra (2010); Binotto, Nakayama e Siqueira (2013), tratam de "transferência de conhecimento"; Tonet e Paz (2006) usam a expressão "compartilhamento de conhecimento"; Vanti (2002) aborda o tema "difusão de conhecimento"; e o mesmo ocorre com inúmeros outros autores e publicações. Porém, apesar da diversidade de nomenclaturas, o conceito implícito refere-se sempre ao mesmo significado. Na presente pesquisa o termo adotado foi "compartilhamento de conhecimento".

O compartilhamento de conhecimento é considerado de suma importância organizacional por Tonet e Paz (2006), embora seja um processo de difícil concretização. Segundo Binotto, Nakayama e Siqueira (2013), o compartilhamento de conhecimento acontece do nível individual para o coletivo, organizacional e entre organizações. E, tão importante quanto compartilhar conhecimentos, experiências e informações, é necessário que estes sejam utilizados na prática, para que produzam resultados concretos.

No meio organizacional o compartilhamento de conhecimento no ambiente de trabalho é uma forma de assegurar que os empregados repassem uns aos outros o conhecimento que possuem garantindo a disseminação e aquisição do conhecimento de que podem necessitar futuramente, em situações similares. A capacidade que determinada organização tem de otimizar a reutilização do conhecimento - que pode, muitas vezes, ficar restrito a alguns indivíduos ou áreas, enquanto os demais podem estar com problemas que poderiam ser solucionados com o conhecimento já dominado internamente - é um relevante diferencial competitivo (TONET; PAZ, 2006).

De acordo com Cunha e Ferreira (2011), existem fatores que facilitam o sucesso do compartilhamento do conhecimento, tais como a seleção adequada dos coordenadores e participantes das equipes envolvidas, apoio da organização, estabelecimento de programas de viagens, visitas, reuniões e outras atividades de socialização, que tendem a motivar os participantes e apoiar o desenvolvimento de relações de confiança entre as equipes. Por outro lado, os mesmos autores ressaltam que existem barreiras no processo de compartilhamento de conhecimento, como a resistência à mudança, fluxo unidirecional de conhecimento e falta de valorização do conhecimento local.

Complementando o exposto por Cunha e Ferreira (2011) a respeito das barreiras existentes no fluxo de conhecimento, Kurtz, Forcellini e Varvakis (2014) acrescentam aspectos como baixa confiabilidade, baixa capacidade de retenção de conhecimento, relutância em aceitar, baixa capacidade de absorção de conhecimento e o não compartilhamento de práticas e informações por receio de perder espaço, considerando-as barreiras críticas presentes no processo de compartilhamento de conhecimento. 
Além disso, Ferreira, Li e Serra (2010) sugerem que o sucesso do compartilhamento de conhecimento depende, entre outros fatores relativos à cultura organizacional, do formato organizacional da empresa, bem como do alinhamento entre a estratégia e a estrutura organizacional, e do sistema de recompensas em prática. De acordo com as necessidades e atores envolvidos no processo, a geração, compartilhamento e uso de conhecimento constituem atividades fundamentais para a inovação, além de mostrarem-se como um desafio para os gestores, uma vez que é necessário saber compartilhar o conhecimento, para que não fique concentrado apenas em algumas pessoas (NONAKA; TAKEUCHI, 1997; VEDOVELLO; FIGUEIREDO, 2005).

Da mesma forma que Oliveira et al. (2013) descrevem a falta de consenso entre o conceito de Gestão do Conhecimento, Tonet; Paz (2006, p. 03) afirmam que apesar de o senso comum identificar facilmente o que seria compartilhar conhecimento, ainda não existe um consenso empírico sobre o significado do constructo. Porém, as autoras apresentam uma definição para compartilhamento de conhecimento como sendo "o comportamento do indivíduo de repassar o que sabe a pessoas com quem trabalha e de receber o conhecimento que elas possuem. A conseqüência esperada desse comportamento é que o destinatário ou receptor assimile o conhecimento compartilhado pela fonte ou emissor".

\section{Procedimentos metodológicos}

A base de dados escolhida para elaboração desse artigo foi a Scopus, considerada uma das principais referências internacionais em pesquisa pela sociedade científica brasileira. A referida base indexa páginas web de conteúdo científico, títulos acadêmicos revisados por pares, séries de livros, entre outros. A mesma oferece funcionalidades de apoio à análise de resultados, além de ferramentas para identificar autores e analisar citações, tratando-se da maior base de dados bibliográfica internacional disponível atualmente (COORDENAÇÃO DE APERFEIÇOAMENTO DE PESSOAL DE NÍVEL SUPERIOR - CAPES, 2014).

Para a realização da presente pesquisa, inicialmente foram feitas buscas no site http://www-scopus-com.ez46.periodicos.capes.gov.br/, inserindo o termo "transmissão de conhecimento". Foi selecionada a opção de busca pelo termo no "título, resumo e palavras-chave", em documentos do tipo "artigo" e período de publicação entre 1994 e 2014, adicionados ao Scopus nos últimos sete dias. A área de interesse selecionada foi "Ciências Sociais e Humanas". Buscando o termo "transmissão de conhecimento" nenhum artigo foi encontrado, havendo "zero resultados".

Em seguida o procedimento foi repetido substituindo o termo "transmissão de conhecimento" por "transferência de conhecimento", utilizando exatamente os mesmos critérios que no passo anterior. Buscando por "transferência de conhecimento" foram encontrados seis resultados, ou seja, seis artigos tratando do assunto. Finalmente, fez-se a busca com o termo "compartilhamento de conhecimento" ao invés de 
"transmissão de conhecimento" ou "transferência de conhecimento". Dessa vez três resultados (artigos referentes ao assunto) foram encontrados. A última busca foi com a expressão "difusão de conhecimento" que não teve nenhum resultado encontrado.

Apenas nove resultados, no total, foram encontrados nessa busca. Então, optou-se por ampliar a pesquisa na base de dados SciELO, que, similarmente à Scopus, é considerada uma das principais bases de dados científicas no ambiente acadêmico brasileiro. A biblioteca eletrônica SciELO (Scientific Electronic Library Online) abrange uma coleção selecionada de relevantes periódicos científicos brasileiros, buscando o desenvolvimento de uma metodologia comum que possibilite preparar, armazenar, disseminar e avaliar a produção científica em formato eletrônico (SciELO, 2014).

Como o objetivo deste estudo foi fazer uma bibliometria dos artigos que tratam do tema compartilhamento de conhecimento, considerou-se que nove artigos não teriam a representatividade necessária para a realização de um estudo bibliométrico, que consiste no mapeamento da quantidade de produção científica sobre determinado assunto, a fim de mensurar a produtividade científica em determinado período de tempo (VANTI, 2002). Então, as bases de dados pesquisadas nessa pesquisa foram SciELO e Scopus.

Para a busca na base de dados SciELO, repetiu-se os critérios e a ordem de pesquisa da busca realizada na Scopus. Após acessar o site http://www.scielo.br/, clicou-se em "pesquisa de artigos", e foi inserido o termo "transmissão de conhecimento", com a orientação de pesquisar no campo "palavras do título e resumo". Nessa busca dois resultados (artigos) foram encontrados.

Posteriormente, o procedimento foi repetido substituindo o termo "transmissão de conhecimento" por "transferência de conhecimento", utilizando os mesmos critérios que no passo anterior. Buscando por "transferência de conhecimento" nenhum artigo foi encontrado. O mesmo aconteceu ao buscar "difusão de conhecimento", pois não houve resultado algum na base de dados em questão. Finalmente, com o termo "compartilhamento de conhecimento", um resultado foi encontrado.

Uma nova pesquisa foi feita seguindo o mesmo padrão anteriormente descrito nas duas bases de dados em questão, adicionando-se o termo "bibliometria" às buscas. Ou seja, buscou-se "compartilhamento de conhecimento" e bibliometria; "transmissão de conhecimento" e bibliometria; "difusão de conhecimento" e bibliometria; e, "transferência de conhecimento" e bibliometria em "todos os índices". No entanto, resultado algum foi encontrado, tanto na SciELO quanto na Scopus.

Os 12 artigos encontrados (no total) foram analisados na etapa de apresentação e análise de dados, bem como na elaboração da fundamentação teórica do presente estudo. Além disso, outros materiais como artigos e livros diversos foram utilizados para elaborar a fundamentação teórica. Esse estudo pode ser considerado quali- 
quantitativo, pois utiliza técnicas tanto de pesquisa quantitativa quanto qualitativa.

A pesquisa quantitativa está presente no uso da bibliometria, que é uma técnica quantitativa de mensuração. A pesquisa quantitativa, segundo Gerhardt e Silveira (2009), é influenciada pelo positivismo e considera que só é possível compreender a realidade se os dados brutos forem analisados, sendo previamente coletados através de instrumentos padronizados e neutros. A pesquisa qualitativa/ funcionalista, que também foi utilizada nesse estudo, é descritiva, com análise indutiva dos dados, sem uso de técnicas e/ou métodos estatísticos (CRESWELL, 2010).

A presente pesquisa classifica-se como bibliométrica, descritiva, indutiva, aplicada e bibliográfica, pois faz uso de conhecimentos já sistematizados, além de descrever o objeto de estudo e as características do mesmo, utilizando material já publicado (ALMEIDA, 2011; GIL, 1999). Essa pesquisa possui dados secundários que foram coletados através de buscas nas bases de dados SciELO e Scopus.

A Revisão de Literatura foi realizada por meio de uma abordagem Integrativa, que por ser um método de revisão mais amplo, permite a inclusão de literatura tanto teórica quanto empírica e estudos com abordagens metodológicas variadas, podendo ser quantitativa e/ou qualitativa (POMPEO; ROSSI; GALVÃO, 2009). A análise e discussão dos dados foi feita de forma descritiva, apresentando os dados encontrados.

\section{Apresentação e análise dos resultados}

Após a coleta dos dados, a próxima etapa foi selecionar informações em comum entre os artigos encontrados e agrupá-los de acordo com as semelhanças. Em seguida foram elaboradas tabelas, a fim de auxiliar na visualização dos resultados encontrados e facilitar a compreensão dos mesmos. As referidas tabelas são apresentadas a seguir. A Tabela 1, por exemplo, trata do número de artigos sobre compartilhamento de conhecimento publicados a cada cinco anos no período pesquisado (1994 - 2014).

Tabela 1 - número de artigos sobre compartilhamento de conhecimento publicados a cada cinco anos

\begin{tabular}{c|c}
\hline Período (1994- 2014) & Total de artigos \\
\hline \hline De 1994 a 1999 & 0 \\
\hline De 2000 a 2004 & 1 \\
\hline De 2005 a 2009 & 2 \\
\hline De 2010 a 2014 & 9 \\
\hline
\end{tabular}

Fonte: Dados da pesquisa.

Observando os dados acerca da quantidade de publicações feitas entre 1994 e 2014 disponibilizadas nas bases de dados SciELO e Scopus pode-se perceber que o número cresceu significativamente a partir do ano 2000. No período de 1994 a 1999 não há registro nessas bases de publicação alguma acerca do tema em questão. O artigo "mais antigo" localizado com a realização dessa pesquisa data do ano 2004 e, desde 
então, o número de publicações sobre o tema tem aumentado rapidamente. É interessante destacar que o número de publicações acerca de compartilhamento de conhecimento e Gestão do Conhecimento teve um aumento expressivo, principalmente entre 2010 e 2014. De 2000 a 2004 houve apenas uma publicação, que evoluíram para duas entre 2005 e 2009, chegando a nove publicações de 2010 a 2014.

Com base nesse aumento no número de publicações, pode-se inferir que 0 interesse pela Gestão do Conhecimento também aumentou, podendo o aumento de publicações ser um indício e uma consequência desse fato. Técnicas, ferramentas e etapas do processo de Gestão do Conhecimento também tem se destacado no decorrer do tempo. Na Tabela 2, a seguir, são apresentadas algumas informações sobre o termo "compartilhamento de conhecimento".

Tabela 2 - número de artigos publicados com cada termo referente a compartilhamento de conhecimento

\begin{tabular}{c|c}
\hline Termo utilizado & Total de artigos \\
\hline \hline Transferência de conhecimento & 5 \\
\hline Compartilhamento de conhecimento & 5 \\
\hline Transmissão de conhecimento & 2 \\
\hline Disseminação de conhecimento & 0 \\
\hline Transferência e Compartilhamento de & 0 \\
\hline Difusão e disseminação de conhecimento & 0 \\
\hline Transferência de conhecimento e trocas de & 0 \\
\hline Difusão e Compartilhamento de conhecimento & 0 \\
\hline
\end{tabular}

Fonte: Dados da pesquisa.

Conforme previamente apresentado nesse estudo, existem nomenclaturas variadas na literatura que se referem ao processo de compartilhamento de conhecimento. $\mathrm{Na}$ Tabela 2 são apresentadas algumas dessas expressões, notando-se a predominância dos termos "compartilhamento de conhecimento" e "transferência de conhecimento", com cinco artigos cada, localizados por meio da busca dirigida. A expressão "transmissão de conhecimento", teve duas ocorrências, enquanto as demais não foram localizadas na busca em questão. No entanto, destaca-se que podem aparecer referindo-se ao mesmo assunto, embora de modo menos numeroso que as expressões "compartilhamento de conhecimento" e "transferência de conhecimento".

Em alguns casos, uma das possíveis causas para a multiplicidade de expressões referindo-se ao mesmo tema pode ser o fato de esses estudos terem sido elaborados com base em publicações internacionais, que consequentemente estão escritas em outro(s) idioma(s), que não o português. Quanto ao idioma utilizado para redigir e apresentar os artigos encontrados na busca realizada no presente estudo, todos os 12 artigos foram escritos em português. Além de informações a respeito do idioma e do ano das publicações, também buscou-se informações a respeito do método utilizado nos estudos em questão, as quais são apresentadas a seguir, na Tabela 3.

Tabela 3 - método utilizado 


\begin{tabular}{c|c}
\hline Método & Total de artigos que o utilizam \\
\hline \hline Quali-quantitativo & 2 \\
\hline Quantitativo & 2 \\
\hline Qualitativo & 8 \\
\hline
\end{tabular}

Fonte: Dados da pesquisa.

Do total de 12 artigos encontrados na presente pesquisa, dois utilizaram 0 método quali-quantitativo e também dois 0 método quantitativo. Contudo, mais da metade dos artigos encontrados utilizaram o método qualitativo, sendo este método utilizado em oito artigos, que correspondem a $67 \%$ do total de artigos encontrados. Algumas informações mais detalhadas referentes aos instrumentos de coleta de dados utilizados são apresentadas na Tabela 4.

Tabela 4 - instrumento de coleta de dados utilizado

\begin{tabular}{c|c}
\hline Instrumento de coleta de dados utilizado & Total de artigos \\
\hline \hline Entrevista & 4 \\
\hline Questionário & 3 \\
\hline Survey & 1 \\
\hline Pesquisa bibliográfica & 1 \\
\hline Entrevistas + análise de documentos + & 1 \\
\hline Observação participativa + questionário & 1 \\
\hline Entrevista + análise de documentos &
\end{tabular}

Fonte: Dados da pesquisa.

Quanto à etapa de coleta de dados, há uma variedade de instrumentos disponíveis que podem ser utilizados para a realização da mesma. No estudo em questão, nota-se a predominância da utilização de questionários - em três artigos - e entrevistas - em quatro artigos. A pesquisa bibliográfica, por sua vez, foi utilizada em um artigo, do mesmo modo que survey, que teve uma única ocorrência.

Além disso, a união de técnicas múltiplas para a coleta de dados foi utilizada em três artigos, que foram: entrevistas + análise de documentos + observação; observação participativa + questionário; entrevista + análise de documentos. Existem diversas outras combinações possíveis que podem ser utilizadas para unir ferramentas, de acordo com a necessidade do pesquisador.

Considera-se que os assuntos tratados nos estudos em questão também constituem elemento relevante a ser considerado na análise dos mesmos. Desse modo, os assuntos abordados nos 12 trabalhos em questão foram analisados e constatou-se que há predominância por publicações tratando do assunto "transferência de conhecimento em empresas multinacionais", uma vez que quatro dos trabalhos analisados tratam dessa temática.

No entanto, os demais temas não apresentam relação explícita entre si, ocorrendo isoladamente. Apesar disso, nota-se interesse significativo pelos Recursos Humanos por parte das organizações. Os temas abordados são: transferência reversa de conhecimento; desperdícios e barreiras ao fluxo de conhecimento; retenção das habilidades; conhecimento tácito; software colaborativo: aspectos individuais e usabilidade; pensamento 
sistêmico; aspectos do espaço físico de trabalho; compartilhamento do conhecimento e inovação.

Nesse estudo analisou-se, ainda, qual a fonte de origem das 12 publicações estudadas nesse artigo. As referidas informações são apresentadas na Tabela 5, a seguir.

Tabela 5 - fonte de origem das publicações

\begin{tabular}{c|c}
\hline Nome do periódico/ evento & Número total de artigos \\
\hline \hline Espacios & 6 \\
\hline Cadernos CEDES & 1 \\
\hline Gestão \& Produção & 1 \\
\hline Revista Brasileira de Gestão e Desenvolvimento Regional & 1 \\
\hline Revista de Administração Contemporânea & 1 \\
\hline Perspectivas em Ciência da Informação & 1 \\
\hline Acta Botanica Brasilica & 1 \\
\hline
\end{tabular}

Fonte: Dados da pesquisa.

Com relação aos periódicos nos quais os artigos em questão foram publicados, a Revista "Espacios" abrange metade dos artigos publicados no período analisado acerca do assunto pesquisado, com seis do total de 12 artigos publicados. Os outros periódicos identificados foram: "Cadernos CEDES"; "Gestão \& Produção"; "Revista Brasileira de Gestão e Desenvolvimento Regional"; "Revista de Administração Contemporânea"; "Perspectivas em Ciência da Informação"; "Acta Botanica Brasilica", que tiveram, cada um, uma publicação sobre o tema nesse período.

Ponderando-se as áreas dos periódicos que abrangem a maioria das publicações sobre o tema em questão, pode-se afirmar que as Ciências Sociais Aplicadas, especialmente Ciências da Informação e Administração parecem ter maior interesse pela Gestão do Conhecimento. É possível que uma das causas de maior interesse e presença nessas áreas seja o fato de que é fundamental para as empresas reterem conhecimentos a fim de aumentar a competitividade e consequentemente, a lucratividade e "longevidade" das mesmas.

\section{Considerações finais}

Com base nos dados obtidos com a realização desse estudo pode-se inferir que Gestão do Conhecimento e compartilhamento de conhecimento são temas relativamente recentes, uma vez que as publicações a respeito começaram a acontecer de forma expressiva a partir de 2009. Os temas recebem, gradativamente, atenção de gestores e pesquisadores de diversas áreas, principalmente Ciências da Informação e Administração.

As publicações em português representaram a totalidade dos 12 artigos encontrados na busca, o que pode sugerir que no Brasil há uma tendência de aumento do número de organizações que adotam essa prática (compartilhamento de conhecimento). A respeito dos métodos empregados pelos autores que publicaram sobre o tema, nota-se que o método qualitativo é o mais utilizado, estando presente em $67 \%$ das publicações. Além disso, os instrumentos de coleta de dados mais 
utilizados são questionários e entrevistas. Vale destacar, ainda, que há possibilidades de utilizar instrumentos como questionários e entrevistas tanto exclusivamente quanto unidos a outros (técnicas múltiplas) num mesmo estudo.

Conforme apresentado anteriormente, os estudos sobre o tema em questão têm crescido rapidamente e nota-se uma tendência de que essa prática se tornará parte da rotina das organizações. Tanto no meio acadêmico quanto organizacional os debates e estudos relacionados à Gestão do Conhecimento estão cada vez mais presentes e valorizados, sendo considerados uma necessidade para manter e aumentar a competitividade, através da manutenção do capital intelectual/ ativos intangíveis, podendo oferecer inúmeras vantagens às organizações que fazem uso da mesma (EVERS, 2001; TONET; PAZ, 2006; CUNHA; FERREIRA, 2011; FREIRE et al., 2012; BINOTTO; NAKAYAMA; SIQUEIRA, 2013).

Pode-se destacar que, para as organizações, é positivo haver compartilhamento de conhecimento, pois além de evitar gastos com retrabalho e refazer treinamentos, evita-se também a repetição de erros, pois os funcionários mais experientes repassam seus conhecimentos e vivências para os demais. Além disso, a união de conhecimentos é mais valiosa que a existência de diversos conhecimentos individuais (TONET; PAZ, 2006; RAMOS; HELAL, 2010; CUNHA; FERREIRA, 2011; FREIRE et al., 2012; LEMOS; JOIA, 2012).

\section{Referências}

ALMEIDA, M. de S. Elaboração de projeto, TCC, dissertação e tese: uma abordagem simples, prática e objetiva. São Paulo: Atlas, 2011.80 p.

BINOTTO, E.; NAKAYAMA, M. K.; SIQUEIRA, E. S. A criação de conhecimento para a gestão de propriedades rurais no Brasil e na Austrália. RESR, Piracicaba-SP, v. 51, n. 4, p. 681-698, out./dez. 2013.

COORDENAÇÃO DE APERFEIÇOAMENTO DE PESSOAL DE NÍVEL SUPERIOR (CAPES). Scopus. 2014. Disponível em:

$<$ http://periodicos.capes.gov.br/?option=com_pcollection\&mn=70\&smn= 79\&cid=63>. Acesso em: 12 jun. 2015.

CRESWELL, J. W. Projeto de pesquisa: métodos qualitativo, quantitativo e misto. 3. ed. Porto Alegre: Artmed, 2010. p. 206-237.

CUNHA, A. J. M.; FERREIRA, M. A. T. Transferência de conhecimento em empresas multinacionais estudo de caso na indústria de papel. Perspectivas em Ciência da Informação, v. 16, n. 4, p. 95-118, out./dez. 2011.

EVERS, H.-D. Towards a Malaysian Knowledge Society. In: INTERNATIONAL

MALAYSIAN STUDIES CONFERENCE, 3., 2001, Bangi. Anais... Bangi: MSC. 2001. 
FERREIRA, M. P.; LI, D.; SERRA, F. A. R. Transferência internacional de conhecimento na multinacional: quando o jogo competitivo multimercado se sobrepõe aos mecanismos internos de coordenação. RAE - Revista de Administração de Empresas, v. 9, n. 1, p. 1-29, jan./jun. 2010.

FREIRE, P. de S. et al. Memória organizacional e seu papel na Gestão do Conhecimento. RCA - Revista de Ciências da Administração, Florianópolis, SC, v. 14, n. 33, p. 41-51, ago. 2012. Disponível em: <https://periodicos.ufsc.br/index.php/adm/article/view/21758077.2012v14n33p41/22535>. Acesso em: 26 maio 2015.

GERHARDT, T. E.; SILVEIRA, D. T. (Orgs). Métodos de pesquisa. Porto Alegre: UFRGS, 2009. 120 p. Disponível em: <http://www.ufrgs.br/cursopgdr/downloadsSerie/derad005.pdf>. Acesso em: 12 abr. 2015.

GIL, A. C. Métodos e técnicas de pesquisa social. São Paulo: Atlas, 1999.

KURTZ, D. J.; FORCELLINI, F. A.; VARVAKIS, G. O pensamento enxuto aplicado ao processo de transferência de conhecimento entre organizações: associação entre desperdícios e barreiras ao fluxo de conhecimento em uma cadeia produtiva. Revista Espacios, v. 35, n. 2, p. 10-18, 2014.

LEMOS, B.; JOIA, L. A. Fatores relevantes à transferência de conhecimento tácito em organizações: um estudo exploratório. Gestão \& Produção, São Carlos, v. 19, n. 2, p. 233-246, 2012.

NONAKA, I.; TAKEUCHI, H. Criação de conhecimento na empresa: como as empresas japonesas geram a dinâmica da inovação. Rio de Janeiro: Campus, 1997.

OLIVEIRA, P. H. de et al. Um estudo sobre Gestão do Conhecimento e inovação em uma empresa multinacional do setor de fast-food: o caso da Subway. Perspectivas em Ciência da Informação, v. 18, n. 1, p. 86-105, jan./mar. 2013.

POMPEO, D. A.; ROSSI, L. A.; GALVAO, C. M. Revisão integrativa: etapa inicial do processo de validação de diagnóstico de enfermagem. Acta Paulista de Enfermagem, São Paulo, v. 22, n. 4, p. 434-438, 2009. Disponível em: <http://www.scielo.br/pdf/ape/v22n4/a14v22n4.pdf>. Acesso em: 12 maio 2015.

RAMOS, É. N. P.; HELAL, D. H. A prática da Gestão do Conhecimento em uma empresa familiar do ramo varejista em Minas Gerais (MG): um estudo de caso. Revista de Gestão da Tecnologia e Sistemas de Informação - JISTEM - Journal of Information Systems and Technology Management, v. 7, n. 2, p. 433-452, 2010.

SCIENTIFIC ELECTRONIC LIBRARY ONLINE - SciELO. SCIELO. 2014. Disponível em: <http://www.scielo.br/?lng=pt>. Acesso em: 12 jun. 2015. 
TONET, H. C.; PAZ, M. das G. T. da. Um modelo para o compartilhamento de conhecimento no trabalho. RAC - Revista de Administração Contemporânea, v. 10, n. 2, p. 75-94, abr./jun., 2006.

TORRES, A. A. L.; ZIVIANI, F.; SILVA, S. M. da. Mapeamento de competências: ferramenta para a comunicação e a divulgação científica. TransInformação, Campinas, v. 24, n. 3, p. 191-205, set./dez. 2012.

VANTI, N. Da Bibliometria à Webometria: uma exploração conceitual dos mecanismos utilizados para medir o registro da informação e a difusão do conhecimento. Ciência da Informação, Brasília. v. 31, n. 2, p. 152-162, maio/ago. 2002.

VEDOVELLO, C.; FIGUEIREDO, P. N. Incubadora de inovação: que nova espécie é essa? RAE - Revista de Administração de Empresas, v. 4, n. 1, p. 1-18, jan./jul. 2005. 